\title{
Uniqueness of Quantum Markov Chains Associated with an $X Y$-Model on a Cayley Tree of Order 2
}

\author{
L. Accardi $1^{*}$, F. M. Mukhamedov ${ }^{2 * *}$, and M. Kh. Saburov ${ }^{2 * * *}$ \\ ${ }^{\text {I}}$ CUniversita degli Studi di Roma is Tor Vergata \\ ${ }^{2}$ International Islamic University, Malaysia \\ Received September 1, 2010; in final form, February 17, 2011
}

\begin{abstract}
We propose the construction of a quantum Markov chain that corresponds to a "forward" quantum Markov chain. In the given construction, the quantum Markov chain is defined as the limit of finite-dimensional states depending on the boundary conditions. A similar construction is widely used in the definition of Gibbs states in classical statistical mechanics. Using this construction, we study the quantum Markov chain associated with an $X Y$-model on a Cayley tree. For this model, within the framework of the given construction, we prove the uniqueness of the quantum Markov chain i.e., we show that the state is independent of the boundary conditions.
\end{abstract}

DOI: $10.1134 / \mathrm{S} 0001434611070029$

Keywords: quantum Markov chain, Cayley tree, XY-model, Gibbs state, phase transition, quasiconditional expectation, graph, dynamical system, quasilocal algebra.

\section{INTRODUCTION}

It is well known that Markov random fields have numerous applications to classical probability theory, statistical physics, etc. Therefore, it is natural to expect that quantum analogs of such fields play the same important role as ordinary random fields. The notion of quantum Markov chain was introduced in [1] and was defined in the algebra of quasilocal algebras consisting of infinite tensor products of finitedimensional algebras. At present, quantum Markov chains are a standard tool in solid-state physics, quantum statistical physics, and quantum information theory [2]-[5].

The first attempts at constructing quantum analogs of classical Markov fields appeared in [4], [6][8]. In these papers, the notion of quantum Markov state examined in [9] was generalized. Note that such states constitute a subclass of quantum Markov chains. The definition of quantum Markov states and chains that generalizes all the well-known definitions of the corresponding states and chains was proposed in [10]; moreover, all the existing examples fall within the scope of this definition.

Note that usually a system can be identified with the vertex of a graph if the graph is not isomorphic to $\mathbb{Z}$. An important role in localization is played by the so-called root vertex of the graph, which allows one to construct nontrivial examples of Markov fields. In the papers mentioned above, quantum Markov fields were studied mostly over the integer lattice $\mathbb{Z}^{d}$. In view of the existence of loops in $\mathbb{Z}^{d}$, nontrivial examples of quantum Markov fields over this lattice were not known. It was shown in [11]-[13] that there are numerous examples of classical Markov fields on Cayley trees. And hence to construct nontrivial examples of quantum Markov fields, it is natural to consider them over such trees. It should be noted that quantum Markov chains over Cayley trees were first studied in [14]; similarly, the thermodynamic limit of a VBS-model on a Cayley tree was considered [15]. The notion of Markov property of states defined on a quasilocal algebra over hierarchical trees was introduced in [16]. We have generalized the construction proposed in [9] to the case of trees. This construction defines a "backward" quantum Markov chain. Note that noncommutative extensions of classical Markov fields associated with the Ising and Potts

\footnotetext{
${ }^{*}$ E-mail: accardi@volterra.uniroma2.it

${ }^{* *}$ E-mail: far75m@yandex.ru

**** E-mail: msaburov@gmail.com
} 
models were studied in [17]-[19]. In the classical case, Markov random fields over trees were considered in [11]-[13], [20], [21].

The present paper is a continuation of the study of quantum Markov chains begun in [16]. Nevertheless, in the present paper, we propose another construction (differing from the one in [16]) of quantum Markov chains which corresponds to a "forward" quantum Markov chain. In this construction, a quantum Markov chain is defined as the limit of finite-dimensional states depending on the boundary conditions. A similar construction is widely used in the definition of Gibbs states in classical statistical mechanics. It is well known that, for systems with local interactions, there always exists at least one Gibbs state. However, the system under consideration can admit several different Gibbs measures; in this case, the system is said to have a phase transition. One of the central problems of statistical physics is the determination of the phase transition or of the uniqueness of the Gibbs measure. For Markov random fields, there exist sufficient conditions ensuring the uniqueness of the Gibbs measure (see [22], [23]). One of the important results in classical and quantum theory is that, for a onedimensional spin system with finite interaction radius there is no phase transition, i.e., there exists a unique Gibbs state. However, the existence of a phase transition depends on the dimension of the lattice. For example, for a two-dimensional continuous $X Y$-model, a phase transition exists.

In the present paper, using ideas and methods of Gibbs measure theory, we study a quantum Markov chain associated with an $X Y$-model on a Cayley tree of order 2. From the physical point of view, one can expect the existence of a phase transition for such a model, because the tree under consideration is not one-dimensional. However, in this case, we prove the uniqueness of the quantum Markov chain within the framework of this construction, i.e., we show that the state is independent of the boundary conditions. It should be noted that, for the model under consideration, there exists a phase transition on a Cayley tree of order three (see [26]).

\section{PRELIMINARIES}

A Cayley tree $\Gamma^{k}$ of order $k \geq 1$ is an infinite tree, i.e., a graph without cycles with exactly $k+1$ edges issuing from each vertex. Let $\Gamma^{k}=(L, E)$, where $L$ is the set of vertices of $\Gamma^{k}$ and $E$ is its set of edges. Two vertices $x$ and $y$ are called nearest neighbors if there exists an edge joining them; this edge is denoted by $l=\langle x, y\rangle$. A set of pairs

$$
\left\langle x, x_{1}\right\rangle, \ldots,\left\langle x_{d-1}, y\right\rangle
$$

is called a path from the point $x$ to $y$. The distance $d(x, y)$ between $x$ and $y$ is defined as the number of edges in the shortest path joining the vertices $x$ and $y$. If one edge $\langle x, y\rangle$ of $\Gamma^{k}$ is cut off, then $\Gamma^{k}$ splits into two connected components called semi-infinite Cayley trees with root vertices $x$ and $y$. In what follows, we shall consider only semi-infinite Cayley trees $\Gamma_{+}^{k}=(L, E)$ with root vertex $x^{0}$.

To simplify notation, we introduce the coordinate structure in $\Gamma_{+}^{k}$ as follows: to the vertex $x^{0}$ we assign the coordinate (0); to the nearest neighbors $x^{0}$ we assign the coordinates $(i)$, where $i$ denotes the $i$ th vertex (the vertices are numbered from right to left). Further, each vertex $x$ of the tree $\Gamma_{+}^{k}$ has coordinates

$$
\left(i_{1}, \ldots, i_{n}\right), \quad \text { where } i_{m} \in\{1, \ldots, k\}, \quad 1 \leq m \leq n .
$$

Thus, the symbol $(0)$ denotes the level 0 , while the vertices $\left(i_{1}, \ldots, i_{n}\right)$ constitute the $n$th level (i.e., $\left.d\left(x^{0}, x\right)=n\right)$ of the tree. Denote

$$
\begin{gathered}
W_{n}=\left\{x \in L: d\left(x, x_{0}\right)=n\right\}, \quad \Lambda_{n}=\bigcup_{k=0}^{n} W_{k}, \quad \Lambda_{[n, m]}=\bigcup_{k=n}^{m} W_{k}, \quad n<m, \\
E_{n}=\left\{\langle x, y\rangle \in E: x, y \in \Lambda_{n}\right\}, \quad \Lambda_{n}^{\mathrm{c}}=\bigcup_{k=n}^{\infty} W_{k} .
\end{gathered}
$$

For $x \in \Gamma_{+}^{k}, x=\left(i_{1}, \ldots, i_{n}\right)$, we set

$$
S(x)=\{(x, i): 1 \leq i \leq k\}
$$


here $(x, i)$ indicates $\left(i_{1}, \ldots, i_{n}, i\right)$. This set is called the set of direct descendants of $x$.

We can easily see from these definitions that

$$
\Lambda_{m}=\Lambda_{m-2} \cup\left(\bigcup_{x \in W_{m-1}}\{x \cup S(x)\}\right), \quad E_{m} \backslash E_{m-1}=\bigcup_{x \in W_{m-1}} \bigcup_{y \in S(x)}\{\langle x, y\rangle\} .
$$

For each vertex $x \in L$, for the algebra of observables $\mathscr{B}_{x}$, we consider the algebra of complex $d \times d$ matrices $M_{d}$. The algebra of observables localized on a finite volume $\Lambda \subset L$ is defined by

$$
\mathscr{B}_{\Lambda}=\bigotimes_{x \in \Lambda} \mathscr{B}_{x}
$$

If $\Lambda^{1} \subset \Lambda^{2} \subset L$, then, as usual, $\mathscr{B}_{\Lambda^{1}}$ is identified with the subalgebra $\mathscr{B}_{\Lambda^{2}}$. Further, by $\mathscr{B}_{\Lambda,+}$ we denote the positive part of $\mathscr{B}_{\Lambda}$. The complete algebra $\mathscr{B}_{L}$ on a Cayley tree is obtained by passing to the inductive limit

$$
\mathscr{B}_{L}=\overline{\bigcup_{\Lambda_{n}} \mathscr{B}_{\Lambda_{n}}}
$$

Further, $\mathscr{S}\left(\mathscr{B}_{\Lambda}\right)$ denotes the set of states defined on $\mathscr{B}_{\Lambda}$.

Recall [24] that by quasiconditional expectation with respect to a triple $\mathscr{C} \subset \mathscr{B} \subset \mathscr{A}$ of $C^{*}$ algebras with units one means a completely positive unit preserving mapping $\mathscr{E}: \mathscr{A} \rightarrow \mathscr{B}$ satisfying the condition

$$
\mathscr{E}(c a)=c \mathscr{E}(a), \quad a \in \mathscr{A}, \quad c \in \mathscr{C} .
$$

The state $\varphi$ on $\mathscr{B}_{L}$ is called a $f$-quantum d-Markov chain associated with $\left\{\Lambda_{n}\right\}$ if, for each $\Lambda_{n}$, there exists a quasiconditional expectation $\mathscr{E}_{\Lambda_{n}^{\mathrm{c}}}$ with respect to the triple $\mathscr{B}_{\Lambda_{n+1}^{\mathrm{c}}} \subseteq \mathscr{B}_{\Lambda_{n}^{\mathrm{c}}} \subseteq \mathscr{B}_{\Lambda_{n-1}^{\mathrm{c}}}$ and a state $\widehat{\varphi}_{\Lambda_{n}^{\mathrm{c}}} \in \mathscr{S}\left(\mathscr{B}_{\Lambda_{n}^{\mathrm{c}}}\right)$ such that, for any $n \in \mathbb{N}$, the following relations hold:

$$
\begin{gathered}
\widehat{\varphi}_{\Lambda_{n}^{\mathrm{c}}}\left|\mathscr{B}_{\Lambda_{n+1} \backslash \Lambda_{n}}=\widehat{\varphi}_{\Lambda_{n+1}^{\mathrm{c}}} \circ \mathscr{E}_{\Lambda_{n+1}^{\mathrm{c}}}\right| \mathscr{B}_{\Lambda_{n+1} \backslash \Lambda_{n}}, \\
\varphi=\lim _{n \rightarrow \infty} \widehat{\varphi}_{\Lambda_{n}^{\mathrm{c}}} \circ \mathscr{E}_{\Lambda_{n}^{\mathrm{c}}} \circ \mathscr{E}_{\Lambda_{n-1}^{\mathrm{c}}}^{\mathrm{c}} \cdots \circ \mathscr{E}_{\Lambda_{1}^{\mathrm{c}}}
\end{gathered}
$$

in the $*$-weak topology.

Note that here the $f$-quantum $d$-Markov chain $\varphi$ generated by $\mathscr{E}_{\Lambda_{n}^{\mathrm{c}}}$ and $\varphi_{\Lambda_{n}^{\mathrm{c}}}$ is well defined. Indeed, by $(2.2)$, we have

$$
\widehat{\varphi}_{\Lambda_{n}^{\mathrm{c}}} \circ \mathscr{E}_{\Lambda_{n}^{\mathrm{c}}}\left|\mathscr{B}_{\Lambda_{n}}=\widehat{\varphi}_{\Lambda_{n+1}^{\mathrm{c}}} \circ \mathscr{E}_{\Lambda_{n+1}^{\mathrm{c}}} \circ \mathscr{E}_{\Lambda_{n}^{\mathrm{c}}}\right| \mathscr{B}_{\Lambda_{n}}
$$

and, for $a \in \mathscr{B}_{\Lambda}$, for $\Lambda \subset \Lambda_{k}$, we obtain

$$
\lim _{n \rightarrow \infty} \widehat{\varphi}_{\Lambda_{n}^{\mathrm{c}}} \circ \mathscr{E}_{\Lambda_{n}^{\mathrm{c}}} \circ \mathscr{E}_{\Lambda_{n-1}^{\mathrm{c}}} \circ \cdots \circ \mathscr{E}_{\Lambda_{1}^{\mathrm{c}}}(a)=\widehat{\varphi}_{\Lambda_{k}^{\mathrm{c}}} \circ \mathscr{E}_{\Lambda_{k}^{\mathrm{c}}} \circ \mathscr{E}_{\Lambda_{k-1}^{\mathrm{c}}} \circ \cdots \circ \mathscr{E}_{\Lambda_{1}^{\mathrm{c}}}(a)
$$

\section{CONSTRUCTION OF QUANTUM MARKOV CHAINS ON A CAYLEY TREE}

Suppose that to each edge $\langle x, y\rangle \in E$ an operator $K_{\langle x, y\rangle} \in \mathscr{B}_{\{x, y\}}$ is assigned. Further, we wish to define the state on $\mathscr{B}_{\Lambda_{n}}$ with boundary conditions $w_{0} \in \mathscr{B}_{(0),+}$ and $\mathbf{h}=\left\{h_{x} \in \mathscr{B}_{x,+}\right\}_{x \in L}$. To this end, we introduce the following notation:

$$
K_{n}=w_{0}^{1 / 2} \prod_{\{x, y\} \in E_{1}} K_{\langle x, y\rangle} \prod_{\{x, y\} \in E_{2} \backslash E_{1}} K_{\langle x, y\rangle} \cdots \prod_{\{x, y\} \in E_{n} \backslash E_{n-1}} K_{\langle x, y\rangle} \prod_{x \in W_{n}} h_{x}^{1 / 2} ;
$$

here, by definition, we set

$$
\prod_{\{x, y\} \in E_{m} \backslash E_{m-1}} K_{\langle x, y\rangle}:=\prod_{x \in W_{m-1}}\left(K_{\langle x,(x, 1)\rangle} \cdots K_{\langle x,(x, k)\rangle}\right) .
$$

It should be noted that, in this definition, the product satisfies the ordering relationship, i.e., the position of the operators $K_{\langle x,(x, i)\rangle}$ should not be changed, because, in general, they do not commute. 
Let

$$
\mathscr{U}_{n]}=K_{n} K_{n}^{*}
$$

Obviously, $\mathscr{U}_{n]}$ is a positive operator.

For any $\Lambda \subseteq$ fin $L$, let $\operatorname{Tr}_{\Lambda}: \mathscr{B}_{L} \rightarrow \mathscr{B}_{\Lambda}$ denote the partial trace. For brevity and convenience, we set $\operatorname{Tr}_{n]}:=\operatorname{Tr}_{\Lambda_{n}}$.

Thus, we define the positive functional $\varphi_{w_{0}, \mathbf{h}}^{(n, f)}$ on $\mathscr{B}_{\Lambda_{n}}$ by the relation

$$
\varphi_{w_{0}, \mathbf{h}}^{(n, f)}(a)=\operatorname{Tr}\left(\mathscr{U}_{n+1]}\left(a \otimes \mathbb{I}_{W_{n+1}}\right)\right)
$$

for each $a \in \mathscr{B}_{\Lambda_{n}}$. Here

$$
\mathbb{I}_{W_{n+1}}=\bigotimes_{y \in W_{n+1}} \mathbb{I}
$$

Note that $\operatorname{Tr}$ denotes the normalized trace on $\mathscr{B}_{L}$.

In order to define the state $\varphi^{(f)}$ on $\mathscr{B}_{L}$ (i.e., on the infinite volume) with given marginal states $\varphi_{w_{0}, \mathbf{h}}^{(n, f)}$, i.e., $\varphi^{(f)} \Gamma_{\mathscr{B}_{\Lambda_{n}}}=\varphi_{w_{0}, \mathbf{h}}^{(n, f)}$, we must impose some constraints on the boundary condition $\left\{w_{0}, \mathbf{h}\right\}$ so that the functionals $\left\{\varphi_{w_{0}, \mathbf{h}}^{(n, f)}\right\}$ satisfy the matching condition

$$
\varphi_{w_{0}, \mathbf{h}}^{(n+1, f)} \Gamma_{\mathscr{B}_{\Lambda_{n}}}=\varphi_{w_{0}, \mathbf{h}}^{(n, f)} .
$$

It is well known [9] that the $\left\{\varphi_{w_{0}, \mathbf{h}}^{(n, f)}\right\}$ satisfy the matching condition if the sequence $\left\{\mathscr{U}_{n]}\right\}$ is projective with respect to $\operatorname{Tr}_{n]}$, i.e.,

$$
\operatorname{Tr}_{n-1]}\left(\mathscr{U}_{n]}\right)=\mathscr{U}_{n-1]} \quad \text { for all } n \in \mathbb{N} .
$$

Theorem 3.1. Suppose that the boundary conditions $w_{0} \in \mathscr{B}_{(0),+}$ and $\mathbf{h}=\left\{h_{x} \in \mathscr{B}_{x,+}\right\}_{x \in L}$ satisfy the following equalities:

$$
\begin{gathered}
\operatorname{Tr}\left(w_{0} h_{0}\right)=1 ; \\
\operatorname{Tr}_{\{x\}}\left(\prod_{i=1}^{k} K_{\langle x,(x, i)\rangle} \prod_{i=1}^{k} h_{(x, i)} \prod_{i=1}^{k} K_{\langle x,(x, k+1-i)\rangle}^{*}\right)=h_{x} \quad \text { for any } \quad x \in L .
\end{gathered}
$$

Then $\left\{\mathscr{U}_{n]}\right\}$ is a projective sequence. Moreover, there exists a unique state $\varphi_{w_{0}, \mathbf{h}}^{(f)}$ on $\mathscr{B}_{L}$ such that

$$
\varphi_{w_{0}, \mathbf{h}}^{(f)}=w-\lim _{n \rightarrow \infty} \varphi_{w_{0}, \mathbf{h}}^{(n, f)} .
$$

Proof. Let us immediately verify relation (3.6). It follows from (3.1) that

$$
\begin{aligned}
\mathscr{U}_{n]}=w_{0}^{1 / 2} & \prod_{m=1}^{n-1}\left(\prod_{\{x, y\} \in E_{m} \backslash E_{m-1}} K_{\langle x, y\rangle}\right) \prod_{\{x, y\} \in E_{n} \backslash E_{n-1}} K_{\langle x, y\rangle} \prod_{x \in W_{n}} h_{x} \\
& \times\left(\prod_{\{x, y\} \in E_{n} \backslash E_{n-1}} K_{\langle x, y\rangle}\right)^{*} \prod_{m=1}^{n-1}\left(\prod_{\{x, y\} \in E_{n-m} \backslash E_{n-m-1}} K_{\langle x, y\rangle}\right)^{*} w_{0}^{1 / 2} .
\end{aligned}
$$

We know that, for distinct $x$ and $x^{\prime}$ taken from $W_{n-1}$, the corresponding algebras $\mathscr{B}_{x \cup S(x)}$ and $\mathscr{B}_{x^{\prime} \cup S\left(x^{\prime}\right)}$ commute; therefore, from (3.2), we obtain

$$
\begin{aligned}
& \prod_{\{x, y\} \in E_{n} \backslash E_{n-1}} K_{\langle x, y\rangle} \prod_{x \in W_{n}} h_{x}\left(\prod_{\{x, y\} \in E_{n} \backslash E_{n-1}} K_{\langle x, y\rangle}\right)^{*} \\
& =\prod_{x \in W_{m-1}} \prod_{i=1}^{k} K_{\langle x,(x, i)\rangle} \prod_{i=1}^{k} h_{(x, i)} \prod_{i=1}^{k} K_{\langle x,(x, k+1-i)\rangle}^{*}
\end{aligned}
$$

MATHEMATICAL NOTES Vol.90 No.2 2011 
Therefore, using (3.8), we find that

$$
\begin{aligned}
\operatorname{Tr}_{n-1]}\left(\mathscr{U}_{n]}\right)= & w_{0}^{1 / 2} \prod_{m=1}^{n-1}\left(\prod_{\{x, y\} \in E_{m} \backslash E_{m-1}} K_{\langle x, y\rangle}\right) \\
& \times \prod_{x \in W_{n-1}} \operatorname{Tr}_{x}\left(\prod_{i=1}^{k} K_{\langle x,(x, i)\rangle} \prod_{i=1}^{k} h_{(x, i)} \prod_{i=1}^{k} K_{\langle x,(x, k+1-i)\rangle}^{*}\right) \\
& \times \prod_{m=1}^{n-1}\left(\prod_{\{x, y\} \in E_{n-m} \backslash E_{n-m-1}} K_{\langle x, y\rangle}\right)^{*} w_{0}^{1 / 2} \\
= & w_{0}^{1 / 2} \prod_{m=1}^{n-1}\left(\prod_{\{x, y\} \in E_{m} \backslash E_{m-1}} K_{\langle x, y\rangle}\right) \prod_{x \in W_{n-1}} h_{x} \\
& \times \prod_{m=1}^{n-1}\left(\prod_{\{x, y\} \in E_{n-m} \backslash E_{n-m-1}} K_{\langle x, y\rangle}\right)^{*} w_{0}^{1 / 2}=\mathscr{U}_{n-1]} .
\end{aligned}
$$

Similarly, it follows from equality (3.7) that $\operatorname{Tr}\left(\mathscr{U}_{n]}\right)=1$, which implies that each functional $\varphi_{w_{0}, \mathbf{h}}^{(n, f)}$ is a state and, therefore, there exists a limiting state.

Note that if we set $k=1$ and $h_{x}=I$ for all $x \in L$, then we obtain conditional amplitudes introduced in [9].

Theorem 3.2. The state $\varphi_{w_{0}, \mathbf{h}}^{(f)}$ is an $f$-quantum d-Markov chain.

Proof. For brevity, setting

$$
K_{[n-1, n]}=\prod_{\{x, y\} \in E_{n} \backslash E_{n-1}} K_{\langle x, y\rangle}, \quad \mathbf{h}^{(n)}=\prod_{x \in W_{n}} h_{x}
$$

we define the quasiconditional expectation $\mathscr{E}_{\Lambda_{n}^{c}}$ for each $n$ as follows:

$$
\begin{aligned}
\widehat{\mathscr{E}}_{\Lambda_{1}^{\mathrm{c}}}\left(x_{[0}\right) & =\operatorname{Tr}_{[1}\left(K_{[0,1]}^{*} w_{0}^{1 / 2} x_{[0} w_{0}^{1 / 2} K_{[0,1]}\right), \quad x_{[0} \in \mathscr{B}_{\Lambda_{0}^{\mathrm{c}}}, \\
\mathscr{E}_{\Lambda_{k}^{\mathrm{c}}}\left(x_{[k-1}\right) & =\operatorname{Tr}_{[n}\left(K_{[k-1, k]}^{*} x_{[k-1}^{*} K_{[k-1, k]}\right), \quad x_{[k-1} \in \mathscr{B}_{\Lambda_{k-1}^{\mathrm{c}}}, \quad k=1,2, \ldots, n+1 ;
\end{aligned}
$$

here $\operatorname{Tr}_{[n}:=\operatorname{Tr}_{\Lambda_{n}^{c}}$. Then, for any monomial of the form

we have

$$
a_{\Lambda_{1}} \otimes a_{W_{2}} \otimes \cdots \otimes a_{W_{n}}, \quad \text { where } \quad a_{\Lambda_{1}} \in \mathscr{B}_{\Lambda_{1}}, \quad a_{W_{k}} \in \mathscr{B}_{W_{k}}, \quad k=2, \ldots, n,
$$

$$
\begin{aligned}
\varphi_{w_{0}, \mathbf{h}}^{(n, f)} & \left(a_{\Lambda_{1}} \otimes a_{W_{2}} \otimes \cdots \otimes a_{W_{n}}\right) \\
& =\operatorname{Tr}\left(\mathbf{h}^{(n+1)} K_{[n, n+1]}^{*} \cdots K_{[0,1]}^{*} w_{0}^{1 / 2}\left(a_{\Lambda_{1}} \otimes a_{W_{2}} \otimes \cdots \otimes a_{W_{n}}\right) w_{0}^{1 / 2} K_{[0,1]} \cdots K_{[n, n+1]}\right) \\
& =\operatorname{Tr}_{[1}\left(\mathbf{h}^{(n+1)} K_{[n, n+1]}^{*} \cdots K_{[1,2]}^{*} \widehat{\mathscr{E}}_{1}^{\mathrm{c}}\left(a_{\Lambda_{1}}\right) a_{W_{2}} K_{[1,2]} \cdots a_{W_{n}} K_{[n, n+1]}\right) \\
& =\operatorname{Tr}_{[n+1}\left(\mathbf{h}^{(n+1)} \mathscr{\mathscr { E }}_{\Lambda_{n+1}^{\mathrm{c}}} \circ \mathscr{E}_{\Lambda_{n}^{\mathrm{c}}} \circ \cdots \circ \mathscr{E}_{\Lambda_{2}^{\mathrm{c}}} \circ \widehat{\mathscr{E}}_{\Lambda_{1}^{\mathrm{c}}}\left(a_{\Lambda_{1}} \otimes a_{W_{2}} \otimes \cdots \otimes a_{W_{n}}\right)\right) .
\end{aligned}
$$

Therefore, for any $a \in \Lambda \subset \Lambda_{n+1}$ from (3.4), in view of (3.1), (3.2), (3.10), and (3.12), we obtain

$$
\varphi_{w_{0}, \mathbf{h}}^{(n, f)}(a)=\operatorname{Tr}_{[n+1}\left(\mathbf{h}^{(n+1)} \mathscr{E}_{\Lambda_{n+1}^{\mathrm{c}}} \circ \mathscr{E}_{\Lambda_{n}^{\mathrm{c}}} \circ \cdots \circ \mathscr{E}_{\Lambda_{2}^{\mathrm{c}}} \circ \widehat{\mathscr{E}}_{\Lambda_{1}^{\mathrm{c}}}(a)\right)
$$

The fact that $\mathscr{U}_{n}$ is projective, implies relation (2.2) for $\varphi_{w_{0}, \mathbf{h}}^{(n, f)}$; therefore, from (3.13), we obtain the required assertion/statement.

Note that this construction was studied in the one-dimensional case in [25]. 


\section{QUANTUM MARKOV CHAINS ASSOCIATED WITH AN $X Y$-MODEL}

In this section, we prove the uniqueness of the $f$-quantum $d$-Markov chain associated with an $X Y$-model on a Cayley tree. Further in this section, we consider the semi-infinite Cayley tree $\Gamma_{+}^{2}=$ $(L, E)$ of order 2. Consider the $C^{*}$-algebra $\mathscr{B}_{L}$ with $\mathscr{B}_{x}=M_{2}(\mathbb{C})$ for each $x \in L$. As is customary, by $\sigma_{x}^{(u)}, \sigma_{y}^{(u)}, \sigma_{z}^{(u)}$, we denote the Pauli matrices corresponding to a vertex $u \in L$, i.e.,

$$
\sigma_{x}^{(u)}=\left(\begin{array}{cc}
0 & 1 \\
1 & 0
\end{array}\right), \quad \sigma_{y}^{(u)}=\left(\begin{array}{cc}
0 & -i \\
i & 0
\end{array}\right), \quad \sigma_{z}^{(u)}=\left(\begin{array}{cc}
1 & 0 \\
0 & -1
\end{array}\right) .
$$

For each edge $\langle u, v\rangle \in E$, we set

$$
K_{\langle u, v\rangle}=\exp \left\{\beta H_{\langle u, v\rangle}\right\}, \quad \beta>0,
$$

where

$$
H_{\langle u, v\rangle}=\frac{1}{2}\left(\sigma_{x}^{(u)} \sigma_{x}^{(v)}+\sigma_{y}^{(u)} \sigma_{y}^{(v)}\right)
$$

In view of the equalities

$$
H_{\langle u, v\rangle}^{2 m}=H_{\langle u, v\rangle}^{2}=\frac{1}{2}\left(\mathbb{I}-\sigma_{z}^{(u)} \sigma_{z}^{(v)}\right), \quad H_{\langle u, v\rangle}^{2 m-1}=H_{\langle u, v\rangle}, \quad m \in \mathbb{N},
$$

we obtain

$$
K_{\langle u, v\rangle}=\mathbb{I}+\sinh \beta H_{\langle u, v\rangle}+(\cosh \beta-1) H_{\langle u, v\rangle}^{2} .
$$

In order to establish the main result of this section, we must describe all the solutions $\left\{w_{0},\left\{h_{x}\right\}\right\}$ of Eq. (3.7), (3.8). Further, we assume that $h_{x}=h_{y}$ for any $x, y \in W_{n}, n \in \mathbb{N}$. Therefore, we denote $h_{x}^{(n)}:=h_{x}, x \in W_{n}$. Using (4.2), (4.3), we can easily see that $K_{\langle u, v\rangle}=K_{\langle u, v\rangle}^{*}$. Therefore, Eq. (3.8) can be written as follows:

$$
\operatorname{Tr}_{x}\left(K_{\langle x, y\rangle} K_{\langle x, z\rangle} h_{y}^{(n+1)} h_{z}^{(n+1)} K_{\langle x, z\rangle} K_{\langle x, y\rangle}\right)=h_{x}^{(n)} \quad \text { for all } \quad x \in L .
$$

After some calculations, the last equation (4.4) can be reduced to the following system:

$$
\left\{\begin{array}{l}
\left(\frac{a_{11}^{(n+1)}+a_{22}^{(n+1)}}{2}\right)^{2} \cosh ^{4} \beta+a_{12}^{(n+1)} a_{21}^{(n+1)} \sinh ^{2} \beta \cosh \beta=a_{11}^{(n)}, \\
a_{12}^{(n+1)} \frac{a_{11}^{(n+1)}+a_{22}^{(n+1)}}{2} \sinh \beta \cosh \beta(1+\cosh \beta)=a_{12}^{(n)}, \\
a_{21}^{(n+1)} \frac{a_{11}^{(n+1)}+a_{22}^{(n+1)}}{2} \sinh \beta \cosh \beta(1+\cosh \beta)=a_{21}^{(n)}, \\
\left(\frac{a_{11}^{(n+1)}+a_{22}^{(n+1)}}{2}\right)^{2} \cosh ^{4} \beta+a_{12}^{(n+1)} a_{21}^{(n+1)} \sinh ^{2} \beta \cosh \beta=a_{22}^{(n)}
\end{array}\right.
$$

here

$$
h_{x}^{(n)}=\left(\begin{array}{cc}
a_{11}^{(n)} & a_{12}^{(n)} \\
a_{21}^{(n)} & a_{22}^{(n)}
\end{array}\right), \quad h_{y}^{(n+1)}=h_{z}^{(n+1)}=\left(\begin{array}{ll}
a_{11}^{(n+1)} & a_{12}^{(n+1)} \\
a_{21}^{(n+1)} & a_{22}^{(n+1)}
\end{array}\right)
$$

From (4.5), we immediately obtain $a_{11}^{(n)}=a_{22}^{(n)}$ for all $n \in \mathbb{N}$. 
The self-adjointness of $h_{x}^{(n)}$, i.e., $\overline{a_{12}^{(n)}}=a_{21}^{(n)}$ for any $n \in \mathbb{N}$, allows us to reduce system (4.5) to

$$
\left\{\begin{array}{l}
\left|a_{11}^{(n+1)}\right|^{2} \cosh ^{4} \beta+\left|a_{12}^{(n+1)}\right|^{2} \sinh ^{2} \beta \cosh \beta=a_{11}^{(n)}, \\
a_{12}^{(n+1)} a_{11}^{(n+1)} \sinh \beta \cosh \beta(1+\cosh \beta)=a_{12}^{(n)} .
\end{array}\right.
$$

Remark 4.1. Since the operators $h_{x}^{(n)}$ are positive, we conclude that

$$
a_{11}^{(n)} a_{22}^{(n)} \geq\left|a_{12}^{(n)}\right|^{2} \quad \text { for all } n \in \mathbb{N} .
$$

Now let us study the resulting system (4.6). To do this, we define the mapping

$$
f:(x, y) \in \mathbb{R}_{+} \times \mathbb{C} \rightarrow\left(x^{\prime}, y^{\prime}\right) \in \mathbb{R}_{+} \times \mathbb{C}
$$

as follows:

$$
\left\{\begin{array}{l}
\left|x^{\prime}\right|^{2} \cosh ^{4} \beta+\left|y^{\prime}\right|^{2} \sinh ^{2} \beta \cosh \beta=x, \\
x^{\prime} y^{\prime} \sinh \beta \cosh \beta(1+\cosh \beta)=y
\end{array}\right.
$$

here $\beta>0$.

We can easily see that the last equation (4.7) can be reduced to the following one:

$$
\left\{\begin{array}{l}
\left|x^{\prime}\right|^{2} \cosh ^{4} \beta+\left|y^{\prime}\right|^{2} \sinh ^{2} \beta \cosh \beta=x, \\
x^{\prime}\left|y^{\prime}\right| \sinh \beta \cosh \beta(1+\cosh \beta)=|y| .
\end{array}\right.
$$

Therefore, further, we shall consider a dynamical system

$$
f:(x, y) \in \mathbb{R}_{+}^{2} \rightarrow\left(x^{\prime}, y^{\prime}\right) \in \mathbb{R}_{+}^{2}
$$

of the form

$$
\left\{\begin{array}{l}
\left|x^{\prime}\right|^{2} \cosh ^{4} \beta+\left|y^{\prime}\right|^{2} \sinh ^{2} \beta \cosh \beta=x, \\
x^{\prime} y^{\prime} \sinh \beta \cosh \beta(1+\cosh \beta)=y .
\end{array}\right.
$$

In view of Remark 4.1, we consider the dynamical system (4.8) in the domain

$$
\Delta=\left\{(x, y) \in \mathbb{R}_{+}^{2}: x \geq y, x \neq 0\right\} .
$$

In what follows, we shall use the following auxiliary fact.

Lemma 4.2. If $\beta>0$, then

$$
0<\sinh \beta(1+\cosh \beta)<\cosh ^{3} \beta .
$$

The proof can be obtained by direct verification of the last inequality.

Theorem 4.3. Suppose that $f$ is the dynamical system given by equality (4.8). Then the following assertions are valid:

i) there exists a unique fixed point of the mapping $f$ belonging to the domain $\Delta$;

ii) the dynamical system $f$ has no periodic points with period $k, k \geq 2$, in the domain $\Delta$.

Proof. (i) Suppose that $(x, y)$ is a fixed point, i.e.,

$$
\left\{\begin{array}{l}
x^{2} \cosh ^{4} \beta+y^{2} \sinh ^{2} \beta \cosh \beta=x, \\
x y \sinh \beta \cosh \beta(1+\cosh \beta)=y .
\end{array}\right.
$$

Consider two different cases, depending on to the value of $y$. 
Case (a). Suppose that $y=0$. Then

$$
\text { either } x=0 \quad \text { or } \quad x=1 / \cosh ^{4} \beta \text {. }
$$

It follows from the definition of $\Delta$ that only the point $\left(1 / \cosh ^{4} \beta, 0\right)$ belongs to this domain.

Case (b). Now suppose that $y \neq 0$. Then, from (4.9), we obtain

$$
x=\frac{1}{\sinh \beta \cosh \beta(1+\cosh \beta)} ;
$$

therefore, we have

$$
y^{2} \sinh ^{2} \beta \cosh \beta=\frac{\sinh \beta \cosh \beta(1+\cosh \beta)-\cosh ^{4} \beta}{\sinh ^{2} \beta \cosh ^{2} \beta(1+\cosh \beta)^{2}} .
$$

It follows from Lemma 4.2 that

$$
\frac{\sinh \beta \cosh \beta(1+\cosh \beta)-\cosh ^{4} \beta}{\sinh ^{2} \beta \cosh ^{2} \beta(1+\cosh \beta)^{2}}<0,
$$

which contradicts the fact that (4.10) is positive. Thus, there is no fixed point in this case.

Thus, the dynamical system has only one fixed point $\left(1 / \cosh ^{4} \beta, 0\right)$.

(ii) Let us now pass to the periodic points. Suppose that the system has a periodic point $\left(x^{(0)}, y^{(0)}\right)$ with period $k, k \geq 2$, in the domain $\Delta$. This implies the existence of distinct points

$$
\left(x^{(0)}, y^{(0)}\right),\left(x^{(1)}, y^{(1)}\right), \ldots,\left(x^{(k-1)}, y^{(k-1)}\right) \in \Delta
$$

such that

$$
\left\{\begin{array}{l}
\left(x^{(i+1)}\right)^{2} \cosh ^{4} \beta+\left(y^{(i+1)}\right)^{2} \sinh ^{2} \beta \cosh \beta=x^{(i)}, \\
x^{(i+1)} y^{(i+1)} \sinh \beta \cosh \beta(1+\cosh \beta)=y^{(i)},
\end{array}\right.
$$

where $i=0, \ldots, k-1$, i.e., $f\left(x^{(i)}, y^{(i)}\right)=\left(x^{(i+1)}, y^{(i+1)}\right)$; here $x^{(k)}=x^{(0)}, y^{(k)}=y^{(0)}$.

Again consider two cases.

Case (a). Suppose that $y^{(0)} \neq 0$. Then $x^{(i)}, y^{(i)}$ are positive for all values of $i=0, \ldots, k-1$. Therefore, we have

$$
\begin{aligned}
\frac{x^{(i)}}{y^{(i)}} & =\frac{\left(x^{(i+1)} / y^{(i+1)}\right)^{2} \cosh ^{4} \beta+\sinh ^{2} \beta \cosh \beta}{x^{(i+1)} / y^{(i+1)} \sinh \beta \cosh \beta(1+\cosh \beta)} \\
& =\frac{\cosh ^{3} \beta}{\sinh \beta(1+\cosh \beta)} \cdot \frac{x^{(i+1)}}{y^{(i+1)}}+\frac{\sinh \beta}{1+\cosh \beta} \cdot \frac{y^{(i+1)}}{x^{(i+1)}},
\end{aligned}
$$

where $i=0, \ldots, k-1$.

In view of $x^{(i)}, y^{(i)}>0$, for all $i=0, \ldots, k-1$, we obtain

$$
\frac{x^{(i)}}{y^{(i)}}>\frac{\cosh ^{3} \beta}{\sinh \beta(1+\cosh \beta)} \cdot \frac{x^{(i+1)}}{y^{(i+1)}}
$$

for all values of $i=0, \ldots, k-1$.

Then it follows from (4.12) that

$$
\frac{x^{(0)}}{y^{(0)}}>\left(\frac{\cosh ^{3} \beta}{\sinh \beta(1+\cosh \beta)}\right)^{k} \cdot \frac{x^{(0)}}{y^{(0)}} .
$$

But this inequality contradicts the assertion of Lemma 4.2. Therefore, the dynamical system (4.8) has no periodic points of order greater than 2 . 
Case (b). Suppose that $y^{(0)}=0$. The inequality $k \geq 2$ implies $x^{(0)} \neq 1 / \cosh ^{4} \beta$. Therefore, from (4.11), we obtain

$$
y^{(i)}=0 \quad \text { for all } \quad i=0, \ldots, k-1 .
$$

Then, using (4.11) again, we see that

$$
\left(x^{(i+1)}\right)^{2} \cosh ^{4} \beta=x^{(i)} \quad \text { for all } \quad i=0, \ldots, k-1 ;
$$

this yields

$$
x^{(i+1)}=\frac{1}{\cosh ^{2} \beta} \sqrt{x^{(i)}} \quad \text { for all } \quad i=0, \ldots, k-1 .
$$

Therefore, we have

$$
x^{(0)}=\frac{1}{\cosh ^{4} \beta} \sqrt[2^{k+1}]{x^{(0)} \cosh ^{4} \beta} .
$$

Hence we see that either $x^{(0)}=0$ or $x^{(0)}=1 / \cosh ^{4} \beta$, which leads to a contradiction.

Let us now write the dynamical system (4.8) in explicit form. To do this, let us solve system (4.8) for $\left(x^{\prime}, y^{\prime}\right)$. From (4.8), we obtain

$$
\left\{\begin{array}{l}
\left(x^{\prime}\right)^{2} \cosh ^{4} \beta+\left(y^{\prime}\right)^{2} \sinh ^{2} \beta \cosh \beta=x, \\
\left(x^{\prime}\right)^{2}\left(y^{\prime}\right)^{2} \sinh ^{2} \beta \cosh ^{2} \beta(1+\cosh \beta)^{2}=y^{2} .
\end{array}\right.
$$

Setting $\left(x^{\prime}\right)^{2}=u,\left(y^{\prime}\right)^{2}=v$, we find the system

$$
\left\{\begin{array}{l}
u \cosh ^{4} \beta+v \sinh ^{2} \beta \cosh \beta=x, \\
u v \sinh ^{2} \beta \cosh ^{2} \beta(1+\cosh \beta)^{2}=y^{2} .
\end{array}\right.
$$

Hence we can easily see that

$$
v=\frac{x-u \cosh ^{4} \beta}{\sinh ^{2} \beta \cosh \beta}
$$

Using this expression, we find the equation

$$
\cosh ^{5} \beta(1+\cosh \beta)^{2} \cdot u^{2}-x \cosh \beta(1+\cosh \beta)^{2} \cdot u+y^{2}=0 .
$$

Solving this equation for $u$, we see that

$$
u_{ \pm}=\frac{x \pm \sqrt{x^{2}-4 y^{2} \cosh ^{3} \beta /(1+\cosh \beta)^{2}}}{2 \cosh ^{4} \beta} .
$$

From (4.13), we obtain

$$
v_{ \pm}=\frac{x \mp \sqrt{x^{2}-4 y^{2} \cosh ^{3} \beta /(1+\cosh \beta)^{2}}}{2 \sinh ^{2} \beta \cosh \beta} .
$$

Since the point $\left(x^{\prime}, y^{\prime}\right)$ belongs to the domain $\Delta$, it follows that $u$ must be greater than $v$. Thus, the mapping $f: \mathbb{R}_{+}^{2} \rightarrow \mathbb{R}_{+}^{2}$ (see (4.8)) in explicit form is given by the following system:

$$
\left\{\begin{array}{l}
x^{\prime}=\sqrt{\frac{x+\sqrt{x^{2}-4 y^{2} \cosh ^{3} \beta /(1+\cosh \beta)^{2}}}{2 \cosh ^{4} \beta}}, \\
y^{\prime}=\sqrt{\frac{x-\sqrt{x^{2}-4 y^{2} \cosh ^{3} \beta /(1+\cosh \beta)^{2}}}{2 \sinh ^{2} \beta \cosh \beta}} .
\end{array}\right.
$$


Remark 4.4. It follows from (4.14) that the mapping $f$ is well defined if and only if $x$ and $y$ satisfy the inequality

$$
x \geq 2 y \sqrt{\frac{\cosh ^{3} \beta}{(1+\cosh \beta)^{2}}},
$$

i.e., $f$ maps $\Delta$ into itself.

Lemma 4.5. Suppose that $f: \Delta \rightarrow \Delta$ is the dynamical system given by (4.14). If $x$ and $y$ are positive and satisfy (4.15), then $x^{\prime}$ and $y^{\prime}$ are also positive and satisfy the following inequality:

$$
\frac{x^{\prime}}{y^{\prime}}<\frac{\sinh \beta(1+\cosh \beta)}{\cosh ^{3} \beta} \cdot \frac{x}{y} .
$$

Proof. The assumptions of the lemma and system (4.14) immediately imply that $x^{\prime}$ and $y^{\prime}$ are positive. Further, using system (4.14), we obtain

$$
\begin{aligned}
\frac{x^{\prime}}{y^{\prime}} & =\frac{\sinh \beta(1+\cosh \beta)}{\cosh ^{3} \beta} \cdot \frac{x+\sqrt{x^{2}-4 y^{2} \cosh ^{3} \beta /(1+\cosh \beta)^{2}}}{2 y} \\
& <\frac{\sinh \beta(1+\cosh \beta)}{\cosh ^{3} \beta} \cdot \frac{x}{y}
\end{aligned}
$$

as required.

Let us now study the asymptotic behavior of the trajectories of the dynamical system.

Theorem 4.6. Suppose that $f: \Delta \rightarrow \Delta$ is the dynamical system given by (4.14). Then the following assertions are valid:

i) if $y^{(0)}>0$, then the trajectory $\left\{\left(x^{(n)}, y^{(n)}\right)\right\}_{n=1}^{\infty}$ of the point $\left(x^{(0)}, y^{(0)}\right)$ is finite, i.e., there exists a number $N_{0} \in \mathbb{N}$ such that, for all $n \geq N_{0}$, the points $\left(x^{(n)}, y^{(n)}\right)$ do not belong to $\Delta$;

ii) if $y^{(0)}=0$, then the trajectory $\left\{\left(x^{(n)}, y^{(n)}\right)\right\}_{n=1}^{\infty}$ of the point $\left(x^{(0)}, y^{(0)}\right)$ has the following form:

$$
\left\{\begin{array}{l}
x^{(n)}=\frac{\sqrt[2^{n}]{x^{(0)} \cosh ^{4} \beta}}{\cosh ^{4} \beta} \\
y^{(n)}=0
\end{array}\right.
$$

Proof. (i) Let $y^{(0)}>0$. Suppose that the trajectory $\left\{\left(x^{(n)}, y^{(n)}\right)\right\}_{n=1}^{\infty}$ of the point $\left(x^{(0)}, y^{(0)}\right)$ is infinite. This means that the points $\left(x^{(n)}, y^{(n)}\right)$ are well defined for all $n \in \mathbb{N}$. Then, in view of Remark 4.4 and Lemma 4.5, we obtain

$$
\frac{x^{(n)}}{y^{(n)}}<\left(\frac{\sinh \beta(1+\cosh \beta)}{\cosh ^{3} \beta}\right)^{n} \cdot \frac{x^{(0)}}{y^{(0)}}
$$

for all $n \in \mathbb{N}$.

On the other hand, by Remark $4.4, x^{(n)}$ and $y^{(n)}$ satisfy the inequality

$$
\frac{x^{(n)}}{y^{(n)}} \geq 2 \sqrt{\frac{\cosh ^{3} \beta}{(1+\cosh \beta)^{2}}}
$$

for all $n \in \mathbb{N}$. From Lemma 4.2, we obtain

$$
\left(\frac{\sinh \beta(1+\cosh \beta)}{\cosh ^{3} \beta}\right)^{n} \rightarrow 0 \quad \text { as } \quad n \rightarrow \infty
$$

MATHEMATICAL NOTES Vol.90 No.2 2011 
combining this with inequality (4.17), we see that (4.18) does not hold for some number $N_{0} \in \mathbb{N}$, which leads to a contradiction.

(ii) Let $y^{(0)}=0$; then it follows from (4.14) that $y^{(n)}=0$ for all $n \in \mathbb{N}$. Therefore, from (4.14), we obtain

$$
x^{(n)}=\sqrt{\frac{x^{(n-1)}}{\cosh ^{4} \beta}} .
$$

Thus, iterating the last equality, we obtain

$$
x^{(n)} \cosh ^{4} \beta=\sqrt[2^{n}]{x^{(0)} \cosh ^{4} \beta} .
$$

It follows from Theorem 4.6 that Eq. (4.4) has the parametric solution $\left(w_{0}(\alpha),\left\{h_{x}(\alpha)\right\}\right)$, given by the formulas

$$
w_{0}(\alpha)=\left(\begin{array}{cc}
\frac{1}{\alpha} & 0 \\
0 & \frac{1}{\alpha}
\end{array}\right), \quad h_{x}^{(n)}(\alpha)=\left(\begin{array}{cc}
\frac{\sqrt[2^{n}]{\alpha \cosh ^{4} \beta}}{\cosh ^{4} \beta} & 0 \\
0 & \frac{\sqrt[2^{n}]{\alpha \cosh ^{4} \beta}}{\cosh ^{4} \beta}
\end{array}\right)
$$

for all $x \in L$, where $\alpha \in \mathbb{R}_{+}$.

Note that the boundary conditions corresponding to the fixed point of system (4.8), correspond to the value $\alpha_{0}=1 / \cosh ^{4} \beta$ in (4.19). Therefore, in what follows, these operators will be denoted by $w_{0}\left(\alpha_{0}\right)$ and $h_{x}^{(n)}\left(\alpha_{0}\right)$, respectively.

Now consider the states $\varphi_{w_{0}(\alpha), \mathbf{h}(\alpha)}^{(n, f)}$ corresponding to the solutions $\left(w_{0}(\alpha),\left\{h_{x}^{(n)}(\alpha)\right\}\right)$. Using the definition of this state, we obtain

$$
\begin{aligned}
& \varphi_{w_{0}(\alpha), \mathbf{h}(\alpha)}^{(n, f)}(x)=\operatorname{Tr}\left(w_{0}^{1 / 2}(\alpha) \prod_{\{x, y\} \in E_{1}} K_{\langle x, y\rangle} \prod_{\{x, y\} \in E_{2} \backslash E_{1}} K_{\langle x, y\rangle} \cdots \prod_{\{x, y\} \in E_{n+1} \backslash E_{n}} K_{\langle x, y\rangle}\right. \\
& \left.\times \prod_{x \in W_{n+1}} h_{x}^{(n+1)}(\alpha) \prod_{\{x, y\} \in E_{n+1} \backslash E_{n}} K_{\langle x, y\rangle} \cdots \prod_{\{x, y\} \in E_{1}} K_{\langle x, y\rangle} w_{0}^{1 / 2}(\alpha)\right) \\
& =\frac{\left(\sqrt[2^{n+1}]{\alpha \cosh ^{4} \beta}\right)^{2^{n+1}}}{\alpha\left(\cosh ^{4} \beta\right)^{2^{n+1}}} \operatorname{Tr}\left(\prod_{\{x, y\} \in E_{1}} K_{\langle x, y\rangle} \prod_{\{x, y\} \in E_{2} \backslash E_{1}} K_{\langle x, y\rangle} \cdots \prod_{\{x, y\} \in E_{n+1} \backslash E_{n}} K_{\langle x, y\rangle}\right. \\
& \left.\times \prod_{\{x, y\} \in E_{n+1} \backslash E_{n}} K_{\langle x, y\rangle} \cdots \prod_{\{x, y\} \in E_{1}} K_{\langle x, y\rangle}\right) \\
& =\frac{\alpha_{0}^{2^{n+1}}}{\alpha_{0}} \operatorname{Tr}\left(\prod_{\{x, y\} \in E_{1}} K_{\langle x, y\rangle} \prod_{\{x, y\} \in E_{2} \backslash E_{1}} K_{\langle x, y\rangle} \cdots \prod_{\{x, y\} \in E_{n+1} \backslash E_{n}} K_{\langle x, y\rangle}\right. \\
& \left.\times \prod_{\{x, y\} \in E_{n+1} \backslash E_{n}} K_{\langle x, y\rangle} \cdots \prod_{\{x, y\} \in E_{1}} K_{\langle x, y\rangle}\right) \\
& =\operatorname{Tr}\left(w_{0}^{1 / 2}\left(\alpha_{0}\right) \prod_{\{x, y\} \in E_{1}} K_{\langle x, y\rangle} \prod_{\{x, y\} \in E_{2} \backslash E_{1}} K_{\langle x, y\rangle} \cdots \prod_{\{x, y\} \in E_{n+1} \backslash E_{n}} K_{\langle x, y\rangle}\right. \\
& \left.\times \prod_{x \in W_{n+1}} h_{x}^{(n+1)}\left(\alpha_{0}\right) \prod_{\{x, y\} \in E_{n+1} \backslash E_{n}} K_{\langle x, y\rangle} \cdots \prod_{\{x, y\} \in E_{1}} K_{\langle x, y\rangle} w_{0}^{1 / 2}\left(\alpha_{0}\right)\right)
\end{aligned}
$$




$$
=\varphi_{w_{0}\left(\alpha_{0}\right), \mathbf{h}\left(\alpha_{0}\right)}^{(n, f)}(x),
$$

for any $\alpha$. Hence

$$
\varphi_{w_{0}(\alpha), \mathbf{h}(\alpha)}^{(n, f)}=\varphi_{w_{0}\left(\alpha_{0}\right), \mathbf{h}\left(\alpha_{0}\right)}^{(n, f)}
$$

for any $\alpha$.

The definition of an $f$-quantum $d$-Markov chain implies that

$$
\varphi_{w_{0}(\alpha), \mathbf{h}(\alpha)}^{(f)}=\varphi_{w_{0}\left(\alpha_{0}\right), \mathbf{h}\left(\alpha_{0}\right)}^{(f)} .
$$

Thus, we have proved the following theorem.

Theorem 4.7. There exists a unique f-quantum d-Markov chain associated with the model (4.2).

\section{ACKNOWLEDGMENTS}

This work was supported by the grants MOHE no. FRGS0308-91 and MOSTI no. 01-01-08SF0079.

\section{REFERENCES}

1. L. Accardi, "The noncommutative Markovian property," Funktsional. Anal. Prilozhen. 9 (1), 1-8 (1975) [Functional Anal. Appl. 9 (1), 1-7 (1975)].

2. L. Accardi and F. Fidaleo, "Entangled Markov chains," Ann. Mat. Pura Appl. (4) 184 (3), 327-346 (2005).

3. L. Accardi and F. Fidaleo, "Non-homogeneous quantum Markov states and quantum Markov fields," J. Funct. Anal. 200 (2), 324-347 (2003).

4. L. Accardi and F. Fidaleo, "Markov property is recent developments on the quantum Markov property," in Quantum Probability and Infinite Dimensional Analysis, $Q P-P Q$ : Quantum Probab. White Noise Anal., Burg, 2001 (World Sci. Publ., River Edge, NJ, 2003), Vol. 15, pp. 1-19.

5. M. Ohya and D. Petz, Quantum Entropy and its Use, in Texts Monogr. Phys. (Springer-Verlag, Berlin, 1993).

6. L. Accardi and F. Fidaleo, "Quantum Markov fields," Infin. Dimens. Anal. Quantum Probab. Relat. Top. 6 (1), 123-138 (2003).

7. L. Accardi and V. Liebscher, "Markovian KMS-states for one-dimensional spin chains," Infin. Dimens. Anal. Quantum Probab. Relat. Top. 2 (4), 645-661 (1999).

8. V. Liebscher, "Markovianity of quantum random fields in the $\mathscr{B}(\mathscr{H})$ case," in Quantum Probability and Infinite Dimensional Analysis, QP-PQ: Quantum Probab. White Noise Anal., Burg, 2001 (World Sci. Publ., River Edge, NJ, 2003), Vol. 15, pp. 151-159.

9. L. Accardi and A. Frigerio, “Markovian cocycles,” Proc. Roy. Irish Acad. Sec. A 83 (2), 251-263 (1983).

10. L. Accardi, F. Fidaleo, and F. Mukhamedov, "Markov states and chains on the CAR algebra," Infin. Dimens. Anal. Quantum Probab. Relat. Top. 10 (2), 165-183 (2007).

11. C. J. Preston, Gibbs States on Countable Sets, in Cambridge Tracts in Math. (Cambridge Univ. Press, London, 1974), Vol. 68.

12. A. Spătaru, "Construction of a Markov field on an infinite tree," Adv. Math. 81 (1), 105-116 (1990).

13. F. Spitzer, "Markov random fields on an infinite tree," Ann. Probab. 3 (3), 387-398 (1975).

14. L. Affleck, T. Kennedy, E. H. Lieb, and H. Tasaki, "Valence bond ground states in isotropic quantum antiferromagnets," Comm. Math. Phys. 115 (3), 477-528 (1988).

15. M. Fannes, B. Nachtergaele, and R. F. Werner, "Ground states of VBS models on Cayley trees," J. Stat. Phys. 66 (3-4), 939-973 (1992).

16. L. Accardi, H. Ohno, and F. Mukhamedov, "Quantum Markov fields on graphs," Infin. Dimens. Anal. Quantum Probab. Relat. Top. 13 (2), 165-189 (2010).

17. F. M. Mukhamedov, "On von Neumann algebras corresponding to translation-invariant Gibbs states of the Ising model on a Bethe lattice,” Teoret. Mat. Fiz. 123 (1), 88-93 (2000) [Theoret. and Math. Phys. 123 (1), 489-493 (2000)].

18. F. M. Mukhamedov, "On a factor associated with the unordered phase of $\lambda$-model on a Cayley tree," Rep. Math. Phys. 53(1), 1-18(2004).

19. F. Mukhamedov and U. Rozikov, "On Gibbs measures of models with competing ternary and binary interactions and corresponding von Neumann algebras," J. Stat. Phys. 114 (3-4), 825-848 (2004); "On Gibbs measures of models with competing ternary and binary interactions and corresponding von Neumann algebras II," J. Stat. Phys. $119(1-2), 427-446$ (2005). 
20. S. Zachary, "Countable state space Markov random fields and Markov chains on trees," Ann. Probab. 11 (4), 894-903 (1983).

21. S. Zachary, "Bounded, attractive and repulsive Markov specifications on trees and on one-dimensional lattice," Stochastic Process. Appl. 20 (2), 247-256 (1985).

22. R. L. Dobrushin, "Description of a random field by means of conditional probabilities and conditions for its regularity," Teor. Veroyatnost. Primenen. 13 (2), 201-229 (1968).

23. H.-O. Georgii, Gibbs Measures and Phase Transitions, in de Gruyter Stud. Math. (Walter de Gruyter, Berlin, 1988), Vol. 9.

24. L. Accardi and C. Cecchini, "Conditional expectations in von Neumann algebras and a theorem of Takesaki," J. Funct. Anal. 45 (2), 245-273 (1982).

25. L. Accardi, "Cecchini's transition expectations and Markov chains," in Quantum Probability and Applications, IV, Lecture Notes in Math., Rome, 1987 (Springer-Verlag, Berlin, 1989), Vol. 1396, pp. 1-6.

26. F. Mukhamedov, and M. Saburov, "Phase transitions for an $X Y$-model on a Cayley tree of order 3 in a quantum Markov chain scheme (Transitions de phases pour un modèle $X Y$ sur un arbre de Cayley d'ordre trois dans un schema de chaines de Markov quantiques)," C. R. Math. Acad. Sci. Paris 349 (7-8), 425-428 (2011). 